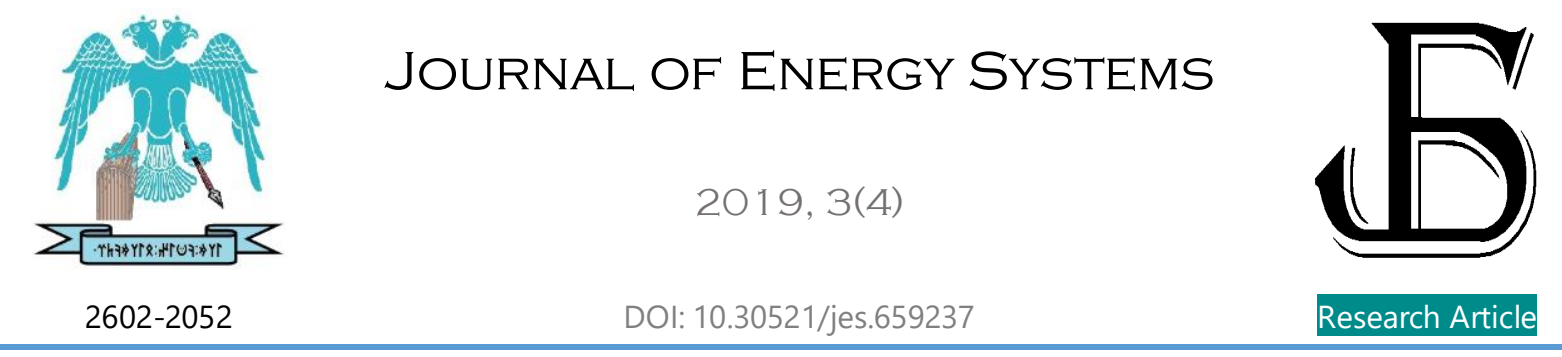

\title{
InP based converter cells under DC plasma influence
}

\author{
Hatice Hilal Kurt ${ }^{(D)}$ \\ Gazi University, Department of Physics, Faculty of Science, Ankara, Turkey, hkurt52tr@gmail.com
}

\begin{tabular}{rr|r|} 
Submitted: & 13.10 .2019 & \\
Accepted: & 26.12 .2019 & \\
Published: & 31.12 .2019 & $\begin{array}{c}\text { Check for } \\
\text { updates }\end{array}$ \\
\hline
\end{tabular}

\begin{abstract}
The plasma parameters have been determined in an infrared image converter for the InP based plasma cell in a broad range of gas pressure from 10 Torr to 760 Torr at room temperature. The electrical properties of the system are controlled by both the plasma and InP electrode. InP has high electron mobility compared to other semiconductors and it can be used for high-speed optoelectronic device applications. Further, any small change in the charge transport mechanism may cause important changes in the system characteristics. The experimental measurements are carried out in air and He media. The homogeneity of the discharge radiation emission depends on the resistivity distribution of the photodetector plate and the radiation intensity is proportional to the plasma current. Local changes in the resistivity of the semiconductor result in local changes in the current and plasma emission.
\end{abstract}

Keywords: Breakdown, DC plasma, InP electrode, Mean electron energy

Cite this paper as:

Kurt, H.H. InP based converter cells under DC plasma influence. Journal of Energy Systems 2019, 3(4), 183-188, DOI: $10.30521 /$ jes. 659237

(c) 2019 Published by peer-reviewed open access scientific journal, JES at DergiPark (https://dergipark.org.tr/en/pub/jes) 


\section{INTRODUCTION}

An electronic bandgap is very important parameter which has responsibility of many optical effects such as electron scattering mechanism by the atoms in a few nanometers scale [1]. The bandgap width has crucial role in the transport mechanism when an external electric field is applied to the material or optically stimulated. InP is a perfect candidate for the optoelectronics systems with plasma connected. Notably, a large research effort has been focused on the alloy semiconductors composed of a plurality of compound semiconductors. Widely known III-V compound semiconductors are GaAs, InP, GaN, and AlN [2]. In those semiconductors, the composition ratio can be changed in a continuous fashion [2]. With these advantages, the III $-\mathrm{V}$ group of materials is well-suited flexible group materials for optoelectronic applications. Therefore, they are efficient materials for solar cells. Flexible spacing features allow the exploration of new designs [3]. InP is an important candidate for optoelectronics systems with plasma connected. InP is widely used in various fields such as flame sensing, medical phototherapy, missile warning, radiation detection, astronomical studies, optical communications and electronic industry [4]. InP is also used for free space laser communications and microwave photonics $[5,6]$. When the voltage is applied to a system, the current is very low at initial stage, which is generated by different sources, such as cosmic rays. By the bombardment of electrons (such as secondary electrons) or collision with gas atoms, a high energy will eventually be generated from the charged particles, thereby increasing the voltage. The current increases when the amount of charged particles is greater, but the output impedance of the power, limits this supply. This stage is commonly referred to as the Townsend discharge zone. In this region, there is generally very small current and uniform electric field distribution along the discharge zone. In this study, the role of gap distance and pressure on the optoelectronic properties of InP have been discussed. The electrical properties of InP wafer are investigated using plasma cell. The plasma currents and gas discharge emissions have been measured with simultaneously in the plasma cell. It is noticed that pressure is very crucial in the transition mechanism from Townsend to Glow phases.

\section{EXPERIMENTAL}

The experimental measurements are carried out in a plasma-semiconductor system with micro gaps lower than $1 \mathrm{~mm}$. Two different gas media are used for working gas at operation process. The plasma chamber is evacuated up to $10^{-1}$ Torr. The electrodes are connected to a power supply system of $2.5 \mathrm{kV}$. The interelectrode distance $d$ is kept on the chosen values to obtain stable discharge conditions. The optimum discharge region both depends on the plasma parameters and the cathode material. For the measurement of the Paschen curves, dc micro discharge system is used with a connected $10 \mathrm{k} \Omega$ series resistor. The gas discharge characteristics of plasma are measured by a multimeter (Keithley 199) and digitalized by custom-made software in a PC. A digital vacuum gate adjusts the discharge pressure for the cell (10 Torr - 760 Torr). The width of the gap is provided by the thickness of the insulating mica foil from $20 \mu \mathrm{m}$ to $770 \mu \mathrm{m}$. 


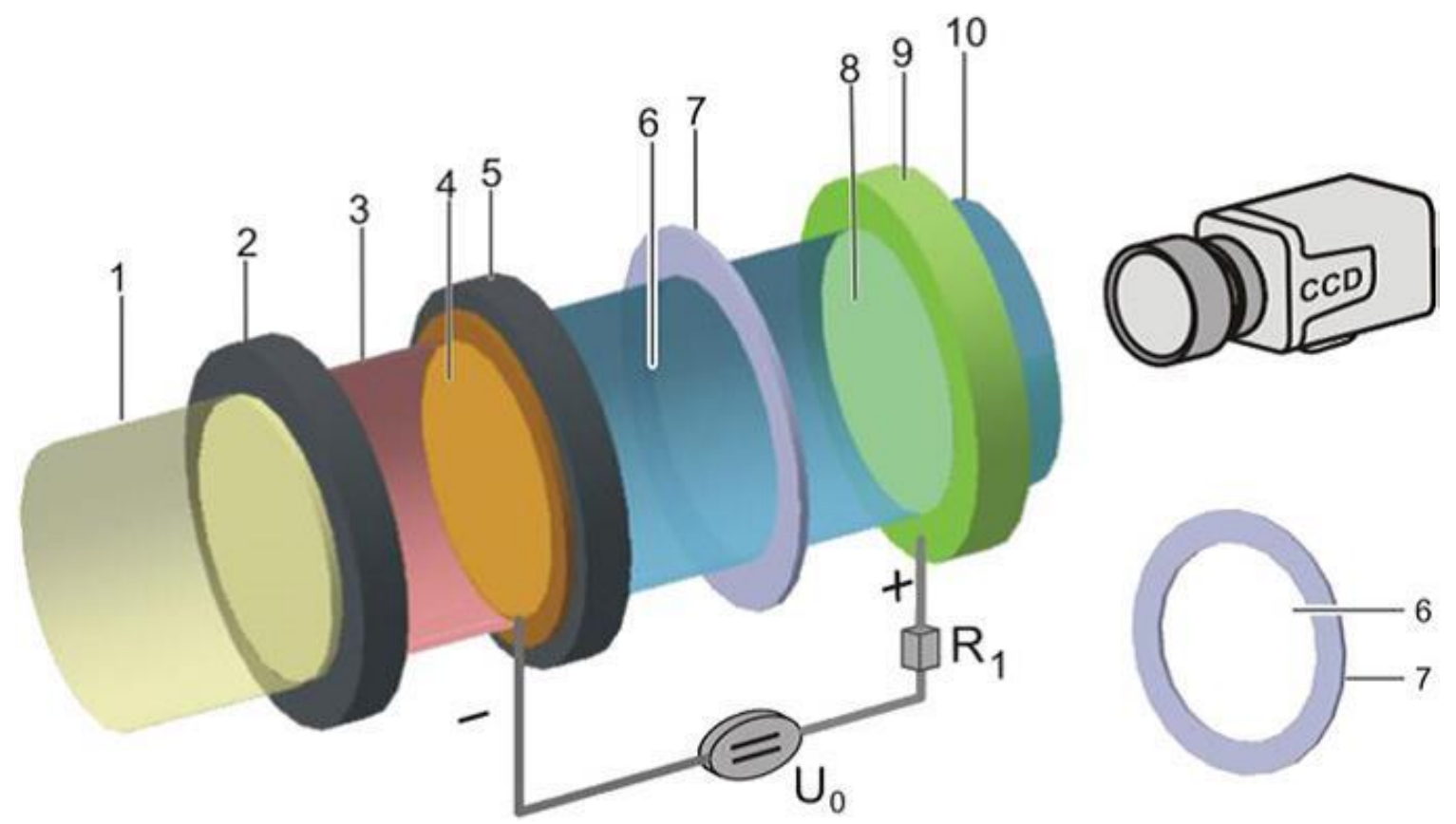

Figure 1. Converter cell with InP cathode : 1-Light beam; 2-Si filter; 3-IR light beam; 4- semitransparent Au contact; 5-InP electrode; 6- The diameter of the gas discharge gap D; 7- insulator mica layer; 8. Semitransparent $\mathrm{SnO}_{2}$ contact; 9-glass disc;10-UV-visible light beam. [7,8]

\section{RESULT AND DISCUSSION}

When the applied voltage on a semiconductor is increased, the electrical conductivity of semiconductor increases because of the production of additional free electrons in the semiconductor due to the increase in the electric field strength. Therefore, the discharge light emission will increase in parallel with the increased electric current [9]. The current and discharge emission emitted from the cell are very sensitive to the changing pressure $\mathrm{p}$ and interelectrode distance.
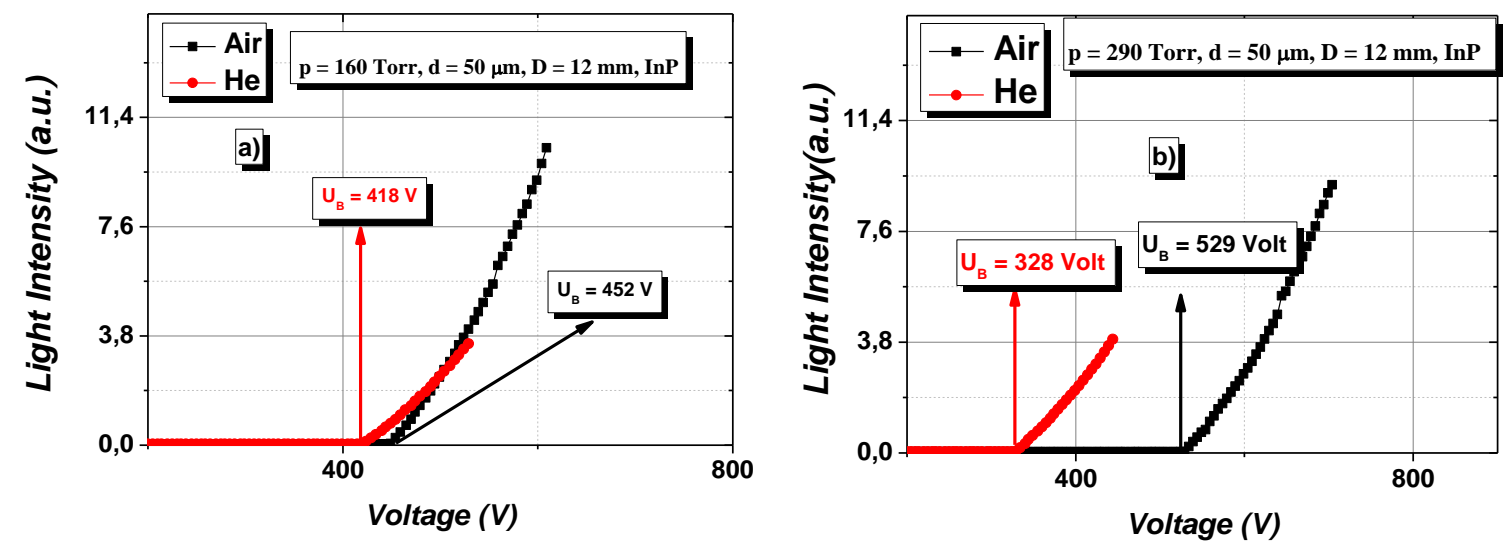

Figure 2. Discharge emission graphs for He and air. a) For $p=160$ Torr. b) For $p=290$ Torr.

When the applied voltage exceeds the breakdown voltage, the self-sustaining discharge is obtained and the gas between the electrodes becomes conductive. The influence of the gas type on the CVCs is studied in the discharge cell with micro gaps. Breakdown occurs earlier for He medium compared to Air. CVCs shows different breakdown process for both gases as seen in Fig.2. The difference between breakdown 
voltages is 30 Volt at 160 Torr, while it is $201 \mathrm{~V}$ at $\mathrm{p}=260$ Torr. However, the effect of gas on CVC is more pronounced at large pressures. The second important point is that the air causes a higher plasma radiation which is approximately twice as high (i.e. 8 (a.u.)).

The simulation of plasma-semiconductor structure plays an important role and finite element analysis software package COMSOL is crucial program to find the plasma parameters such as electron density, mean electron energy, plasma temperature and potential distribution across the plasma cell under the uniform electric field. Plasma has many applications in the field of semiconductor industry, gas cleaning and fusion energy. Plasma consist of the energetic electrons, neutral atoms, excited species, positive ions and negative ions, metastable atoms and free radicals. In its configuration, comsol physics provides modelling and analysis power [10-12] for different areas [10-13].
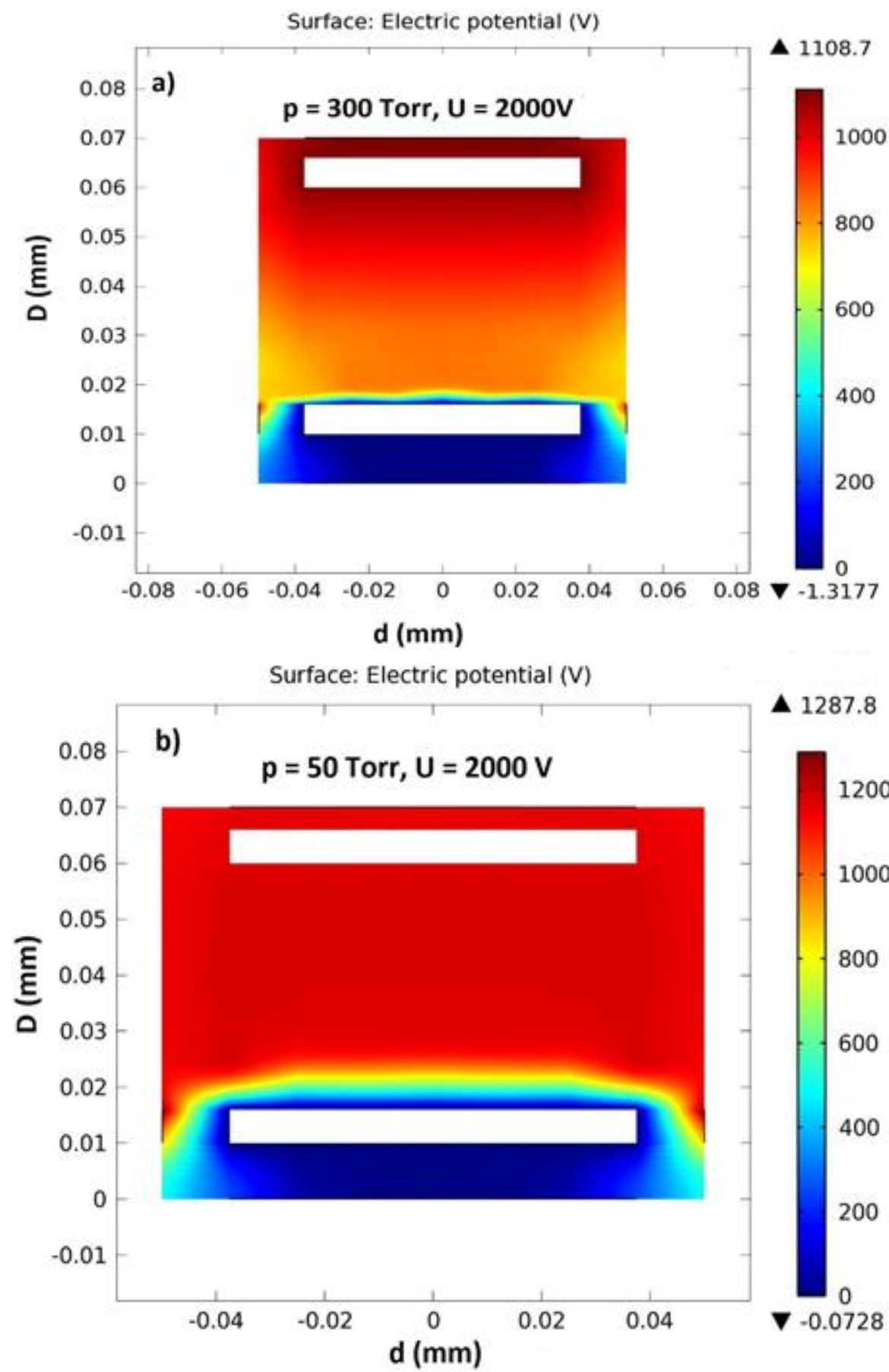

Figure 3. Potential distributions along the active area of plasma. 
In addition, software package COMSOL also contains optional modules inside it. The simulation of the electron potential maps are done for input pressure from 50 Torr - 300 Torr at fixed applied voltage $(2000 \mathrm{~V})$. Potential distributions vary with changing pressure values. Plasma characteristics directly depend on the pressure inside the plasma chamber. Plasma dynamics are defined by the number of the electrons generated during the avalanche mechanism between cathode and anode. Figure 3 a shows that potential value is $1108.7 \mathrm{~V}$ for 300 Torr and $1287.8 \mathrm{~V}$ for 50 Torr.

Mean electron energy distribution is shown in Fig.4. 2D energy distribution shows that electrons are accumulated around the cathode. The main reason is the secondary electron emission from the cathode due to space charge effect. Mean electron energy distribution is obtained for 100 Torr at 2000 Volt. The comprehensive model assumes a uniform electron density and temperature in the discharge space as stated in [8-10] Plasma discharge characteristics are performed by solving particle continuity equations and considering energy conservation. Concerning the simulation result, the electron mean energy is divided into two parts as shown in Figure 4. All electrons are concentrated in the central region of the cathode.

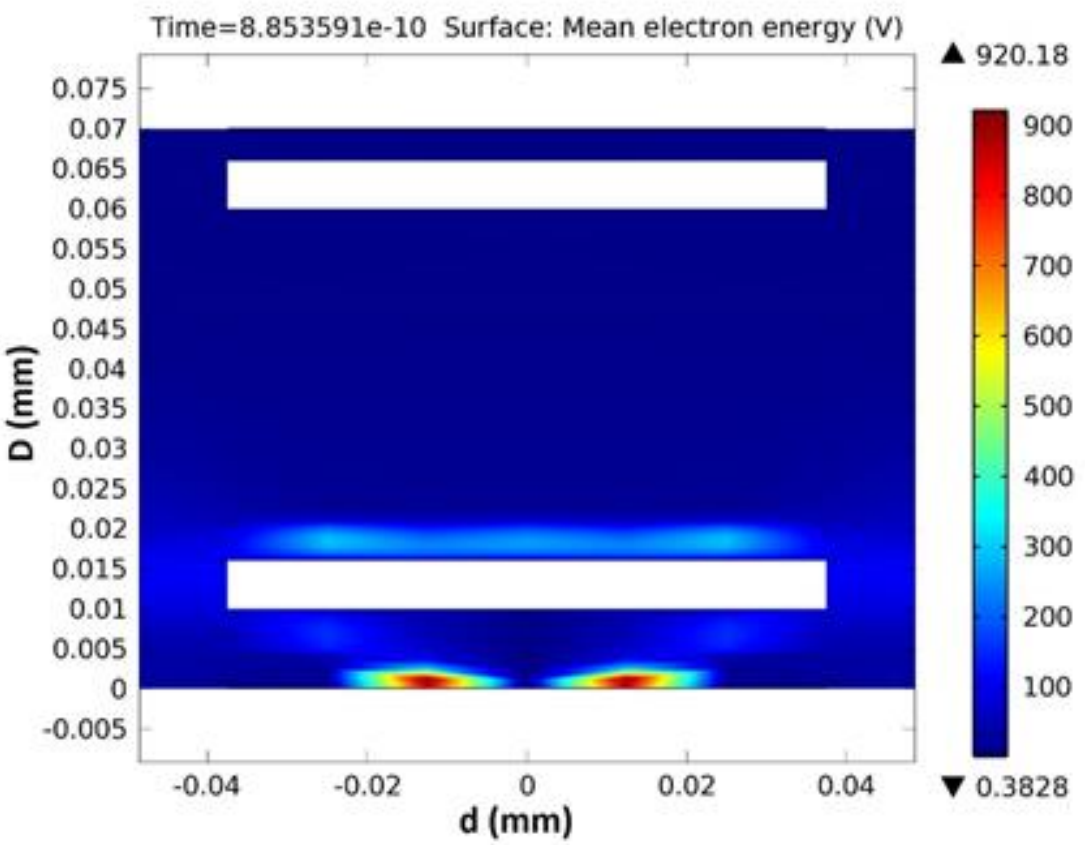

Figure 4. Mean electron energy distribution for $2000 \mathrm{~V}$.

\section{CONLUSION}

The influence of the gas type on the CVCs is studied theoretically and experimentally. The gas influence on the plasma curves are clarified for $\mathrm{He}$ and air environments. In addition, voltage distribution images and mean electron energy are obtained by simulation programme using dc plasma module. The voltage distribution maps take place between the $1108 \mathrm{~V}$ and $1287 \mathrm{~V}$. The density of the electrons depends on the gas pressure and applied voltage in the interelectrode area. Degree of the gas ionization is directly proportional to the selected gas inside the cell. Additional electrons are reached with the positive ions strike in front of the cathode. Those electrons cause the general behavior of the plasma and electron distribution throughout the cell. 


\section{REFERENCES}

[1] Kurt, H.H., \& Salamov, B.G. Breakdown phenomenon and electrical process in a microplasma system with InP electrode, JOM. 2019. DOI: 10.1007/s11837-019-03956-0

[2] Hayasi, H. Development of compound semiconductor devices -In Search of immense possibilities-, SEI Technical Review, 2011, 72, 1-13

[3] Fathpour, S. Emerging heterogeneous integrated photonic platforms on silicon, Nanophotonics, 2015, 4, 143164

[4] Sweeney S.J., Mukherjee J. (2017) Optoelectronic Devices and Materials. In: Kasap S., Capper P. (eds) Springer Handbook of Electronic and Photonic Materials. Springer Handbooks. Springer, Cham

[5] Klamkin, J.et al., Indium phosphide photonic integrated circuits: technology and applications, 2018 IEEE BiCMOS and Compound Semiconductor Integrated Circuits and Technology Symposium (BCICTS), San Diego, CA, 2018, pp. 8-13.DOI: 10.1109/BCICTS.2018.8550947 DOI:

[6] Y. Fu, Zhang, P, Verboncoeur, J. P. Paschen's curve in microgaps with an electrode surface protrusion. Applied Physics Letters 2018, 113, 054102. DOI: 10.1063/1.5045182

[7] Kurt, H.H. Exploration of the infrared sensitivity for a ZnSe electrode of an IR image converter. Journal of Elec Materi. 2018, 47, 4486-4492. DOI: 10.1007/s11664-018-6319-1

[8] Salamov, BG, Kurt, HY, Current instability in a planar gas discharge system with a large-diameter semiconductor cathode. Journal of Physics D: Applied Physics, 2005, 38(5), 682-687.

[9] Kurt, H.H., Tanrıverdi, E. \& Salamov, B.G. Optical and electrical properties of CdS material in a microplasma cell under IR stimulation. JOM. 2019, 71, 644-650. DOI:10.1007/s11837-018-3296-Z

[10] Jia, C. Linhong, J. Kesheng, W. Chuankun, H., Yixiang, S. Two-dimensional simulation of inductively coupled plasma based on COMSOL and comparison with experimental data. Journal of Semiconductors, 2013, 34(6), 066004.

[11] COMSOL Multiphysics Reference Manual, version 5.2, COMSOL, Inc, www.comsol.com.

[12] Divya Deepak, G., Joshi, N. K., Prakash, R., Model analysis and electrical characterization of atmospheric pressure cold plasma jet in pin electrode configuration. AIP Advances, 2018 8, 055321. DOI: $10.1063 / 1.5023072$

[13] Fan Lei, Xiaoping Li, Yanming Liu, Donglin Liu, Min Yang, and Yuanyuan YuFan Lei, Xiaoping Li, Yanming Liu, Simulation of a large size inductively coupled plasma generator and comparison with experimental data, AIP Advances, 2018 8, 015003. DOI: 10.1063/1.5016354 